\title{
Determination of the Refractive Index of Particles Through the Immersion Solid Matching Method
}

\author{
Ilpo Niskanen ${ }^{\circledR}$, Janne Lauri ${ }^{\circledR}$, Member, IEEE, Masayuki Yokota ${ }^{\circledR}$, Rauno Heikkilä ${ }^{\circledR}$, \\ Taro Hashimoto $^{(\mathbb{D}}$, and Tapio Fabritius ${ }^{(\mathbb{}}$, Member, IEEE
}

\begin{abstract}
We describe a new measurement technique to determine the refractive index (RI) of solid particles by monitoring the simultaneous scattering of light from suspension with a camera and the value of the RI with a refractometer. Our immersion solid matching method is based on the solidification process of the liquid continuously changing the RI as a function of time. The principle of the determination is based on finding the RI matching point of the particle and the liquid during the solidification. The traditional liquid immersion method is based on a set of immersion liquids with different refractive indices, while the proposed method requires only a small amount of a single immersion liquid. The method was tested using calcium fluoride $\left(\mathrm{CaF}_{2}\right)$ particles with a varnish immersion liquid. The RI value of $\mathrm{CaF}_{2}$ was obtained to be consistent with the literature values, thus indicating the proper functioning of the proposed procedure.
\end{abstract}

Index Terms-Camera, immersion solid method, particles, refractive index $(\mathrm{RI})$, refractometer, solidification.

\section{INTRODUCTION}

$\mathbf{T}$ HE purpose of advanced materials is to improve the performance of the product by exploiting their unique physical and chemical properties. The global advanced materials market was valued at U.S. 42.76 billion in 2015 and was anticipated to reach U.S. 102.48 billion by 2024 [1]. In material science, the refractive index (RI) is one of the most important and fundamental parameters of matter. Knowing the accurate value of the RI of a material allows the prediction of the photon interaction (reflection, absorption, and scattering) at the interface. This information is especially important in many chemical industries (paints, coatings, cosmetics, pulp, and paper) and photonics. However, the accurate measurement of the RI of powders, which usually have a broad size distribution and present various geometrical shapes, is not an easy problem to be solved. It becomes a challenging issue especially when novel materials are developed.

This work was supported by Business Finland (4294/31/2018) and Academy of Finland's FIRI funding (grant no. 320017). The Associate Editor coordinating the review process was Dr. V. R. Singh. (Corresponding author: Ilpo Niskanen.)

Ilpo Niskanen and Rauno Heikkilä are with the Faculty of Technology, Structures and Construction Technology, University of Oulu, FI-90014 Oulu, Finland (e-mail: ilpo.niskanen@oulu.fi).

Janne Lauri and Tapio Fabritius are with the Faculty of Information Technology and Electrical Engineering, Optoelectronics and Measurement Techniques Research Unit, University of Oulu, FI-90014 Oulu, Finland.

Masayuki Yokota and Taro Hashimoto are with the Department of Mechanical, Electrical and Electronic Engineering, Faculty of Science and Engineering, Shimane University, Shimane 690-8504, Japan.

Digital Object Identifier 10.1109/TIM.2020.3016070
The RI of particles has been investigated using several techniques. Well-known methods include ellipsometry and interferometry. Both methods are accurate (0.0001-0.00001 RI unit), but they are expensive and require samples with very smooth surfaces [2]. Nanoparticle tracking analysis (NTA) is a technique for determining the RI of suspended nanoparticles based on the rate of their Brownian motion [3]. However, that approach is possible only for the nanoparticles composed of high RI materials, such as metals. Surface plasmon resonance (SPR) has been used to determine the RI of nanoparticles, including calcium fluoride [4]. The method is high sensitive even for small sample volumes $(\sim 6 \mathrm{aL})$, but it is time consuming.

The RI of a particle can be determined by an experimental method based on RI matching between the immersion fluid and the particles. This well-known traditional immersion liquid method has been successfully utilized in microscopy [5], spectroscopy [6], and machine vision [7] to determine the RI of particles. In the literature, different variations of the liquid immersion method are described to assess the RI of particles. The principle of these methods is the same, but the matching procedure differs. These methods include the immersion liquid set method, the temperature matching method, the wavelength matching method, the double variation matching method, and the liquid evaporation wavelength matching method [6], [8]. The principle of the immersion liquid method is to find an optimal RI match between the liquid and solid particles embedded in the immersion liquid. Basically, a set of immersion liquid pairs with slightly different RIs is prepared and in the ideal case (the RI of particles match with the RI of immersion liquid), the minimum light scattering $(0 \%)$ is obtained [5]. This approach is relatively simple, and the shape, size, and concentration of the particles do not usually cause significant issues for the accuracy of the determination [2].

In this article, we suggest an alternative approach, the so-called immersion solid detection method, which is based on refractometry and photography imaging. The host liquid, which in this study is varnish, is permitted to dry. Due to the solidification of the liquid, the RI of the liquid continuously increases as a function of the drying time, which is measured by the Abbe refractometer in real time. The Abbe refractometer method is based on the observation of the critical angle of total reflection [9], [10]. During the sweep of the RI, the scattering of light is detected with a camera. The reading of refractometer is associated with corresponding photography images. The occurrence of RI matching between 


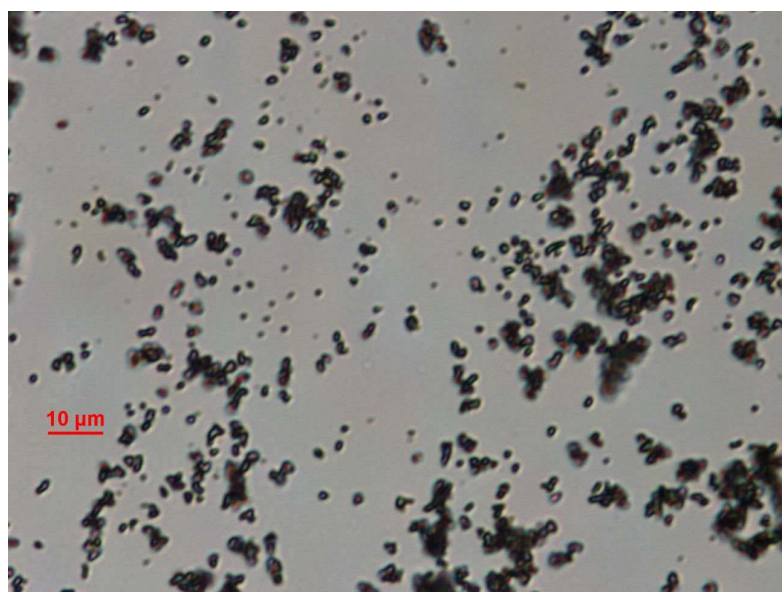

Fig. 1. Light microscopy image of $\mathrm{CaF}_{2}$.

the solidifying host liquid and particles can be observed in captured images as a scattering minimum. The method was tested using calcium fluoride $\left(\mathrm{CaF}_{2}\right)$ particles as a sample. $\mathrm{CaF}_{2}$ finds a wide variety of applications in metallurgical, chemical, and optical materials industries [11]. Therefore, raw material quality is an essential part of process control. The aim of this work was to develop a low-cost and reasonably accurate method for the determination of the RI of particles. The key advantage is that the measurements can be done using only one instrument. This method may lead to a variety of applications being enabled for tailoring and manipulating the optical properties of particles exploited in various applications, such as cosmetics, paint, printing inks, paper, and plastic products.

\section{Materials And Methods}

In this study, Mr. Color H30 Clear varnish (Transparent, by GSI Creos) was used. The varnish is composed of s-butanol (less than 1\%), propyl alcohol (less than 1\%), isopropyl alcohol (20\%-30\%), ethanol (10\%-20\%), isobutyl alcohol (5\%-20\%), ethylene glycol monobutyl ether (1\%-5\%), and ethylene glycol monoethyl ether $(4.9 \%) . \mathrm{CaF}_{2}$ powder was purchased from Merck. The purity of the $\mathrm{CaF}_{2}$ sample was higher than $97 \%$, but according to the product description, it contained small amount of $\mathrm{Fe}, \mathrm{Mg}$, and $\mathrm{SiO}_{4}$. The light microscopy (Eclipse LV100DA-U, Nikon, Japan) method gave an average particle size for $\mathrm{CaF}_{2}$ less than $1 \mu \mathrm{m}$, as shown in Fig. 1.

The solid immersion method layout is shown in Fig. 2. A digital camera was integrated into a refractometer, which makes it possible to measure the scattering of light from the sample container. The light source of the refractometer uses a sodium lamp with a wavelength of $589.6 \mathrm{~nm}$. The reflectometer's light is guided to the surface of the sample and the intensity of the scattering light is monitored by the camera. The room lights were switched off during the measurement and window curtains closed. The camera was fixed at about $6 \mathrm{~cm}$ of distance (minimum focus distance) from a sample container and the measurement area was focused
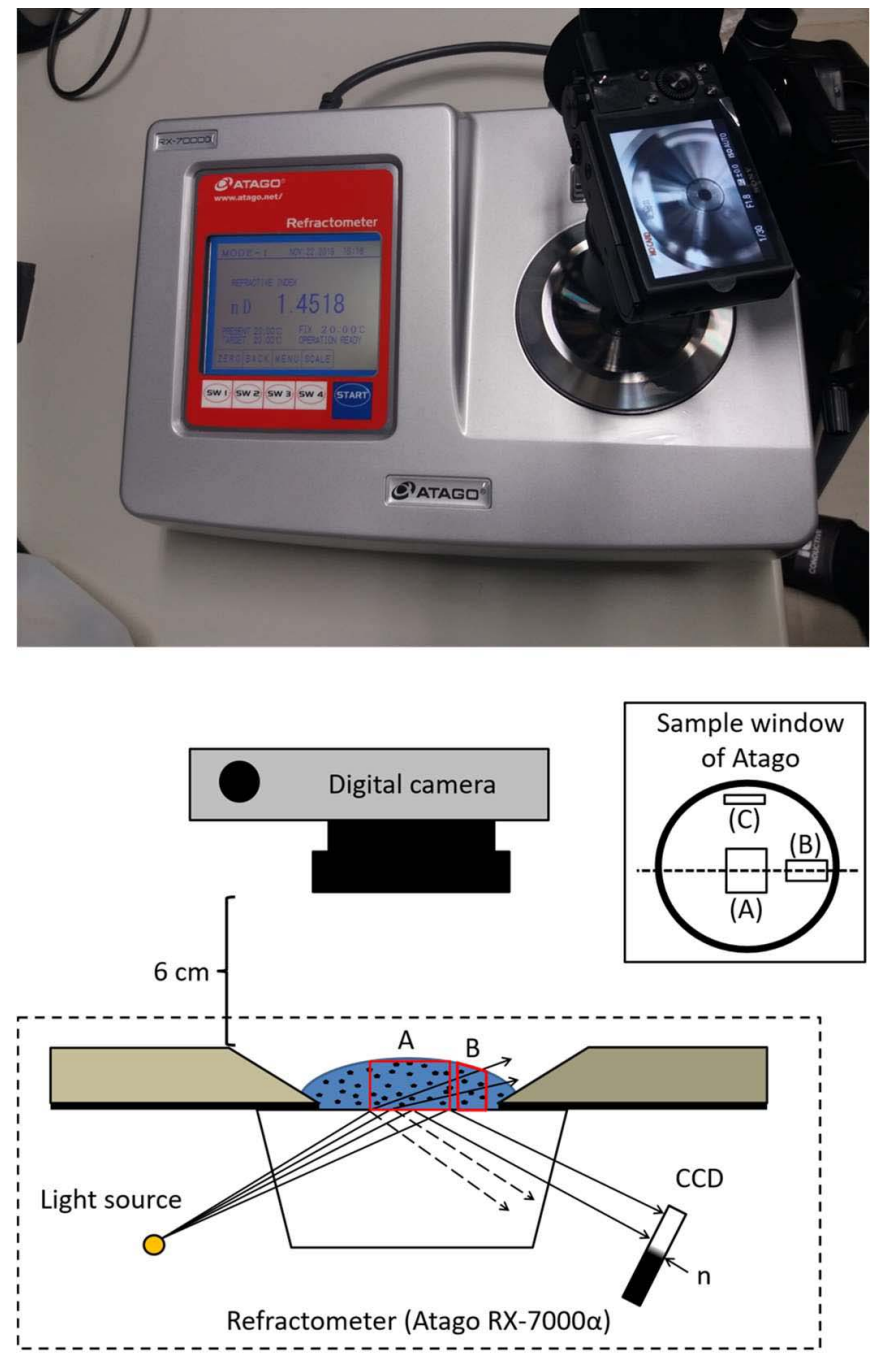

Fig. 2. RI measurement system and the illustration of the measurement principle.

on the sample by manual settings (shutter speed from $1 / 30$, ISO 100, aperture F2).

In this work, a Sony RX 100 camera was used. The RX 100 is equipped with a 20.2-megapixel Exmor CMOS sensor, an F1.8 Zeiss lens, and 3.6x optical zoom. The captured images are saved in an ARW (Sony Alpha Raw) format with a bit depth of 48 bits and a color space of standard redgreen-blue (sRGB). ARW files are converted to 16-bit tiff by Corel Paintshop Pro X9 (Corel Corporation) for further processing in MATLAB (MATLAB R2019a, MathWorks). The image data processing was performed for intensity images, and thus, the RGB color information was converted into the grayscale by the MATLAB built-in function "rgb2gray." The black represents the weakest intensity and the white the strongest intensity [12]. The scattering intensity levels are defined as the mean grayscale intensity calculated inside the rectangular area B shown in Fig. 3(a). The size of the area $B$ is $100 \times 30$ pixels $(1.55 \mathrm{~mm} \times 0.46 \mathrm{~mm})$. In order to compensate the changes of lightning conditions, the obtained scattering intensity levels are divided by the mean reference intensity calculated from the rectangle $\mathrm{C}(100 \times 30$ pixels $)$ in Fig. 3(a). 

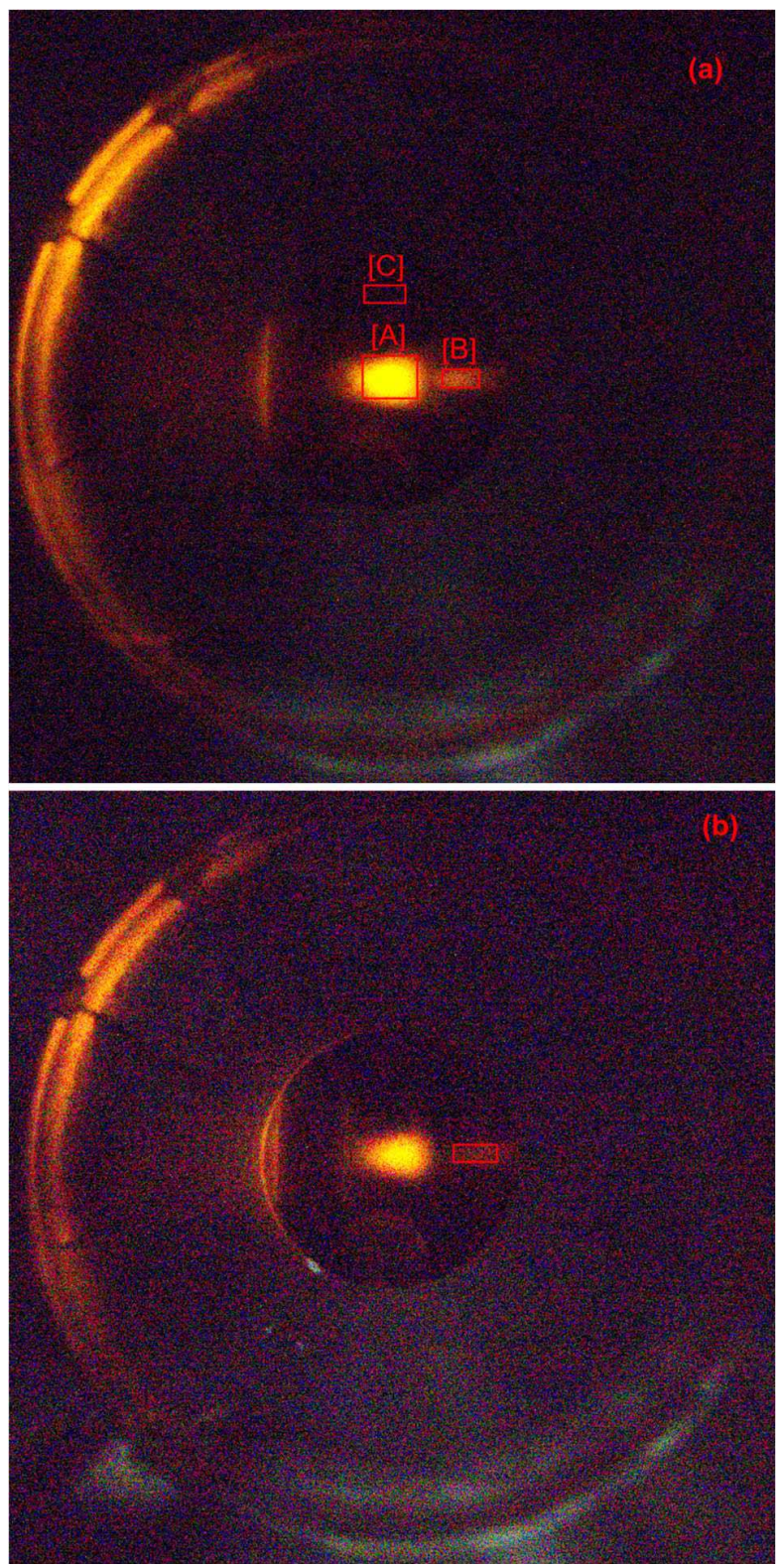

Fig. 3. Images as a function of the $\mathrm{RI} \mathrm{CaF}_{2}$ (a) end ( $\left.n=1.4735,100 \mathrm{~min}\right)$ and (b) match ( $n=1.4346,60 \mathrm{~min})$

\section{RESUlTS AND Discussion}

\section{A. RI Varnish}

Before the actual RI measurement of particles, the RI of the pure varnish was determined using a commercial refractometer (Atago RX-7000 $\alpha$ ) with an average accuracy of \pm 0.0001 refractive units at a regulated constant $\left(21^{\circ} \mathrm{C}\right.$ and $30 \%$ relative humidity). In the beginning ( $0 \mathrm{~min})$ and the end (400 $\mathrm{min})$ of the test, the measured RI values were 1.4056 and 1.4904, respectively. The change in the RI of the varnish as a function of time is depicted in Fig. 4. It shows that the RI of the varnish is nonlinearly depending on the drying time. The reason is that after the paint surface is dried, the evaporation rate of varnish decreases.

It is worth of notice that liquid can contain different types of solvents with a wide range of evaporation rates causing nonlinear drying as a function of time [13]. That is why we

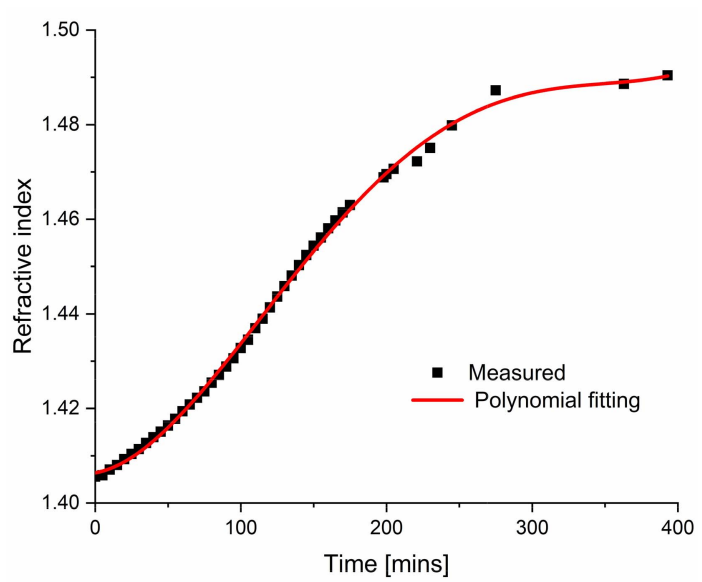

Fig. 4. RI of the varnish as a function of drying process time as measured by the Atago refractometer.

measure the RI of the solidification of the liquid in real time to compensate the drying-based nonlinearity.

\section{B. RI of $\mathrm{CaF}_{2}$}

The applicability of the immersion solid method was tested by measuring the RI of calcium fluoride at $589 \mathrm{~nm}$. The sample suspension consisted of $2 \mathrm{~g}$ of varnish and $0.15 \mathrm{~g}$ of $\mathrm{CaF}_{2}$ powder. The RI of the suspension was measured with the Atago RX-7000 $\alpha$ refractometer at 10 -min intervals. The RI of the suspension was measured to change from 1.41 to 1.47 during the 100-min measurement time. Simultaneously, images (two for every RI value) were recorded from the sample by the camera. The experiments were performed in laboratory room conditions (at a temperature of $22{ }^{\circ} \mathrm{C}$ and a relative humidity of $55 \%$ in dark). The representative examples of obtained photography images from the suspension with two different RI values are shown in Fig. 3. In Fig. 3(a), three rectangular areas (A, B, and $\mathrm{C}$ ), used in RI determination, are depicted at the moment when the RI of suspension is 1.4735 .

The reflected light of area (A) is used to determine the RI of the suspension in the refractometer, while the scattered light in area (B) is utilized to determine the RI of the particle. Area (C) is used as a reference for the scattering measurement. The mean intensity of the grayscale images was determined from the rectangular square area (B) divided by the mean intensity from rectangle $(\mathrm{C})$. The scattering intensity value was determined as an average from two consecutive images taken at the same RI value. Finally, the scattering results for $\mathrm{CaF}_{2}$ were plotted in Fig. 5 as a function of the RI. We can observe from Fig. 5 that $\mathrm{CaF}_{2}$ suspension achieved the minimum scattering at the RI of 1.435 . At this point, the varnish layer with the $\mathrm{CaF}_{2}$ particles becomes nearly transparent, as shown in Fig. 3(b). The transparency is caused by the RI difference matching between the varnish and the particles. The accuracy of the assessment of the RI of the particle from the scattering signal $(y)$ can be improved by using the Lorentzian fitting procedure, which works for particles that have a Lorentzian size distribution

$$
y=y_{0}-\frac{2 A}{\pi} \frac{\omega}{4\left(n_{\text {liquid }}-n_{\text {particle }}\right)^{2}+\omega^{2}}
$$




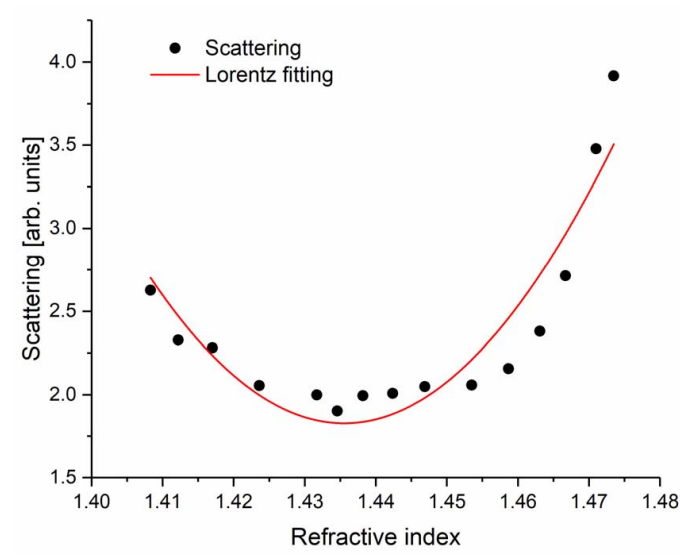

Fig. 5. Scattering data as a function of the RI of an immersion liquid (filled black squares), in this case $\mathrm{CaF}_{2}$ at $589 \mathrm{~nm}$. The solid line is a Lorentzian line used to fit the experimental data.

where $y_{0}$ is the baseline, $A$ is the amplitude, and $\omega$ is the width of the normal distribution at the half-maximum [14]. The Lorentzian function procedure can be applied to scattering and transmission signals, but the amplitude $A$ is negative for scattering and positive for transmission intensities. The fitted Lorentzian curve is shown in Fig. 5. In principle, the minimum of such a function or its zero derivatives can be used in the graphical estimation of the best index match. The best RI estimate was 1.436 for $\mathrm{CaF}_{2}$ at $598 \mathrm{~nm}$. The value of the RI of $\mathrm{CaF}_{2}$ has been reported to be 1.431-1.435 at $589 \mathrm{~nm}$. Kano et al. [4] used the SPR technique, and the RI was measured to be 1.431 at $589 \mathrm{~nm}$. Palik [15] determined the RI by the ellipsometer, resulting in a value of 1.434 . The analysis by Niskanen et al. [16] was based on the immersion liquid matching method which gave the RI as 1.435. Our value of $\mathrm{RI}$ is slightly higher than the literature values at $589 \mathrm{~nm}$.

The results in Fig. 5 show that the minimum point of the scattering curve does not reach a value of one which indicates zero scattering. This is due to the light scattering from the impurities of the $\mathrm{CaF}_{2}$ powder making the RI match imperfect. Another reason could be air bubbles in the sample. The RI mismatch increases the scattering at the best RI matching point, and it might cause a flattening of the scattering curve as well.

The concentrated solid particles of the suspension can scatter light toward the detector, disturbing the specular reflection detection, causing errors in the refractometer measurement. In addition, the varnish drying rate near the open surface could be faster than inside creating a sort of RI gradient inside the measured sample. This phenomenon could be mitigated by using a thin sample layer. The temperature variation might cause some errors because the RI of materials depends on the temperature. For example, RI of water change approximately $0.0001 \mathrm{RI}$ unit for every $1{ }^{\circ} \mathrm{C}$ [6]. Other less significant error sources are the use of a monochromatic light source, a graduated series of calibrated liquids, the particle homogeneity, and the skill of the operator.

\section{CONCLUSION}

In this article, we introduced a laboratory immersion solid method for determining the RI of a particle based on solidification. In this study, varnish is used as an immersion liquid and the RI of $\mathrm{CaF}_{2}$ was determined at $589 \mathrm{~nm}$. The obtained RI value differs by 0.0023 refractive units compared with the early published data. Pigment manufacturers typically provide information about the RI of their products with two-decimal accuracy. The advantage of the proposed method is that only one immersion liquid is needed to determine the RI of the particle with a commercial refractometer and a camera. Since the limited number of liquids is adequate for the immersion-based RI analysis, the decreased number of required solvents is clearly advantageous. In addition, the proposed method is independent of the size, concentration, and shape of the particle.

The solidification time of the suspension tends to be quite long (about $60 \mathrm{~min}$ in our case). An increase in the temperature of the liquid enhances the solidification rate and reduces the time needed for measurement. However, the RI is typically determined at room temperature, which is ca. $20{ }^{\circ} \mathrm{C}$ which means that accelerating the measurement procedure by increasing the temperature would require heating element. Fortunately, many refractometers have an implemented heating unit and a temperature control. The one used in this study can heat up to $70^{\circ} \mathrm{C}$. On the other hand, the measurement time can be influenced by selecting the immersion liquids with faster evaporation properties such as glue.

By combining a camera with a refractometer, a simple and inexpensive device for determining the RI of liquids and solid particles can be implemented. The key point is that the measurements can be done using only one instrument, decreasing the investment costs and simplifying the operational practices such as calibrations and maintenance.

In the future, the method will be used to study the RI of tailored particles and faster solidifying fluids, such as transparent instant adhesives, and to continue the research of dispersion in materials.

\section{REFERENCES}

[1] Advanced Materials (Ceramics, Glasses, Polymers, Composites, and Metals \& Alloys) Market for Medical Devices, Automotive, Aerospace, Electricals \& Electronics, Industrial, Power and Other Applications-Global Industry Analysis, Size, Share, Growth, Trends, and Forecast, 2016-2024, Transparency Market Research. Accessed: Feb. 2016. [Online]. Available: https://www. transparencymarketresearch.com/specialty-silica-market.html

[2] J. G. Webster, The Measurement, Instrumentation and Sensor Handbook. Boca Raton, FL, USA: CRC Press LLC, 1999.

[3] R. Márquez-Islas, C. Sánchez-Pérez, and A. García-Valenzuela, "Determination of refractive index, size, and concentration of nonabsorbing colloidal nanoparticles from measurements of the complex effective refractive index," Opt. Lett., vol. 39, no. 3, pp. 559-562, 2014.

[4] H. Kano, A. Iseda, K. Ohenoja, and I. Niskanen, "Refractive index measurement of nanoparticles by immersion refractometry based on a surface plasmon resonance sensor," Chem. Phys. Lett., vol. 654, pp. 72-75, Jun. 2016.

[5] E. E. El-Hinnawi, Methods in Chemical and Mineral Microscopy. Amsterdam, The Netherlands: Elsevier, 1966, p. 222.

[6] I. Niskanen, K. Hibino, and J. Räty, "Immersion liquid techniques in solid particle characterization: A review," Talanta, vol. 149, pp. 235-236, Mar. 2016.

[7] I. Niskanen, V. Sutinen, G. Thungström, and J. Räty, "Image information by CCD-camera during immersion liquid evaporation process for measuring refractive index of solid particles," Appl Spectrosc., vol. 72, no. 6, pp. 908-912, Feb. 2018. 
[8] S. Wiederseiner, N. Andreini, G. Epely-Chauvin, and C. Ancey, "Refractive-index and density matching in concentrated particle suspensions: A review," Exp. Fluids, vol. 50, no. 5, pp. 1183-1206, Nov. 2010

[9] J. Rheims, J. Köser, and T. Wriedt, "Refractive-index measurements in the near-IR using an Abbe refractometer," Meas. Sci. Technol., vol. 8, no. 6, pp. 601-605, Jun. 1997.

[10] G. H. Meeten and A. N. North, "Refractive index measurement of absorbing and turbid fluids by reflection near the critical angle," Meas. Sci. Technol., vol. 6, pp. 215-221, May 1995.

[11] N. Pawlik, B. Szpikowska-Sroka, T. Goryczka, and W. A. Pisarski, "Solgel glass-ceramic materials containing $\mathrm{CaF}_{2}: \mathrm{Eu}^{3+}$ fluoride nanocrystals for reddish-orange photoluminescence applications," Appl. Sci., vol. 9, no. 24 , p. 5490 , Dec. 2019 , doi: $10.3390 /$ app9245490.

[12] U. M. Jalal, S. C. Kim, and J. S. Shim, "Histogram analysis for smartphone-based rapid hematocrit determination," Biomed. Opt. Express, vol. 8, no. 7, p. 3317, 2017.

[13] I. Niskanen et al., "Monitoring drying process of varnish by immersion solid matching method," Prog. Organic Coat., vol. 136, Nov. 2019, Art. no. 105299.

[14] P. G. Weidler and F. Friedrich, "Determination of the refractive index of particles in the clay and sub-micrometer size range," Amer. Mineralogist, vol. 92, no. 7, pp. 1130-1132, Jul. 2007.

[15] E. D. Palik, Handbook of Optical Constants of Solids II. San Diago, CA, USA: Academic, 1998, pp. 825-881.

[16] I. Niskanen, J. Räty, and K.-E. Peiponen, "Optical sensing of concentration and average refractive index of pigments in a suspensions," Appl. Opt., vol. 49, no. 17, pp. 3428-3433, Jun. 2010, doi: 10.1364/AO. 49.003428 .

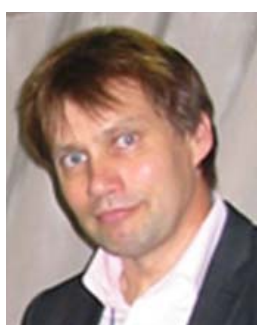

Ilpo Niskanen received the M.Sc. degree in electrical engineering from the University of Oulu, Oulu, Finland, in 2000 and the Ph.D. degree in physics (applied optics) from the University of Joensuu, Joensuu, Finland, in 2008.

He was a Post-Doctoral Researcher of measurement of optical properties index of solid by X-ray holography with the National Institute of Advanced Industrial Science and Technology, Tsukuba, Japan, from 2014 to 2015 and determination of optical properties of nanocellulose with Department of Electronics Design, Mid Sweden University, Sundsvall, Sweden, from 2017 to 2018. Since 2018, he has been an Adjunct Professor of particle characterization techniques and imaging technologies in autonomous work machines at the Faculty of Technology, University of Oulu. He has authored 35 peer-reviewed articles in journals. His current research interests include sensor technology, optical, spectroscopy, optical properties of materials, calculation algorithms, and image analysis.

Janne Lauri (Member, IEEE) received the M.Sc. and D.Sc. (Tech.) degrees in engineering from the University of Oulu, Oulu, Finland, in 2007 and 2013 , respectively.

$\mathrm{He}$ is currently a Post-Doctoral Researcher with the Optoelectronics and Measurement Techniques Research Unit, University of Oulu. His current research interests include optical coherence tomography, microfluidics, flow dynamics of industrial suspensions, and the development of optical imaging methods for printed intelligence manufacturing.

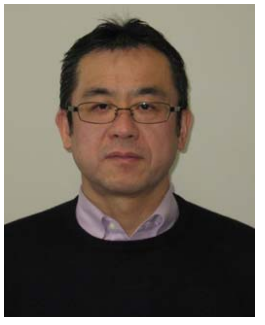

Masayuki Yokota was born in Gunma, Japan, on January 31, 1967. He received the B.S., M.S., and $\mathrm{Ph} . \mathrm{D}$. degrees in electronic engineering from Gunma University, Maebashi, Japan, in 1989, 1991, and 1998, respectively.

$\mathrm{He}$ is currently a Professor with the Department of Mechanical, Electrical, and Electronic Engineering, Shimane University, Matsue, Japan, where he has been working on optical engineering.

Dr. Yokota is a member of the Optical Society of Japan (OSJ), the Imaging Society of Japan (ISJ), the International Society of Optical Engineering (SPIE), and the Optical Society of America (OSA).

Rauno Heikkilä received the Dr. Tech. degree from the University of Oulu, Oulu, Finland, in 1996.

$\mathrm{He}$ is currently a Professor of digitalized construction and mining operations with the Faculty of Technology, University of Oulu, where he is the Head of the Structures and Construction Technology Research Unit. His major research interests cover structures and construction technology, engineering mechanics, information modeling (building information modeling (BIM) in construction) automation and robotics in the construction of roads, railways, fairways, bridges, and buildings as well as in mining. His current research interests include digitalized construction and mining operations, BIM, construction automation and robotics, mining automation and robotic, autonomous machines, and vehicles.

Taro Hashimoto received the B.Sc. degree in electronic engineering from Shimane University, Matsue, Japan, in 2018, where he is currently pursuing the M.Sc. degree.

His current research interests include signal processing, holography technology, industrial suspensions, and imaging methods.
Tapio Fabritius (Member, IEEE) received the M.Sc. and D.Sc. degrees in applied electronics engineering from the University of Oulu, Oulu, Finland, in 2003 and 2007, respectively.

He has been with the University of Oulu since 2003, where he is currently a Full Professor and the Head of the Optoelectronics and Measurement Techniques Research Unit. He has authored over 60 peer-reviewed articles in reputed journals. His current research interests include the development of instrumentation and printed intelligence manufacturing technologies. 